University of Nebraska - Lincoln

DigitalCommons@University of Nebraska - Lincoln

M. Eugene Rudd Publications

Research Papers in Physics and Astronomy

$11-1994$

\title{
Binary-Encounter-Dipole Model for Electron-Impact Ionization
}

Yong-Ki Kim

National Institute of Standards and Technology, Gaithersburg, Maryland

M. Eugene Rudd

University of Nebraska - Lincoln, erudd@unl.edu

Follow this and additional works at: https://digitalcommons.unl.edu/physicsrudd

Part of the Physics Commons

Kim, Yong-Ki and Rudd, M. Eugene, "Binary-Encounter-Dipole Model for Electron-Impact lonization" (1994). M. Eugene Rudd Publications. 34.

https://digitalcommons.unl.edu/physicsrudd/34

This Article is brought to you for free and open access by the Research Papers in Physics and Astronomy at DigitalCommons@University of Nebraska - Lincoln. It has been accepted for inclusion in M. Eugene Rudd Publications by an authorized administrator of DigitalCommons@University of Nebraska - Lincoln. 


\title{
Binary-encounter-dipole model for electron-impact ionization
}

\author{
Yong-Ki Kim \\ National Institute of Standards and Technology, Gaithersburg, Maryland 20899 \\ M. Eugene Rudd \\ Department of Physics and Astronomy, University of Nebraska-Lincoln, Lincoln, Nebraska 68588-0111
}

(Received 6 May 1994)

\begin{abstract}
A theoretical model, which is free of adjustable or fitted parameters, for calculating electronimpact ionization cross sections for atoms and molecules is presented. This model combines the binary-encounter theory with the dipole interaction of the Bethe theory for fast incident electrons. The ratios of the contributions from distant and close collisions and interference between the direct and exchange terms are determined by using the asymptotic behaviors predicted by the Bethe theory for ionization and for stopping cross sections. Our model prescribes procedures to calculate the singly differential cross section (energy distribution) for each subshell using the binding energy, average kinetic energy, and the differential dipole oscillator strengths for that subshell. Then the singly differential cross section is integrated over the ejected electron energy to obtain the total ionization cross section. The resulting total ionization cross section near the threshold is proportional to the excess energy of the projectile electron. We found that this model yields total ionization cross sections for a variety of atoms and molecules from threshold to several $\mathrm{keV}$ which are in good agreement $(\sim 10 \%$ or better on average) with known experimental results. The energy distributions also exhibit the expected shapes and magnitudes. We offer a simpler version of the model that can be used when differential oscillator strengths are not known. For the ionization of ions with an openshell configuration, we found that a minor modification of our theory greatly improves agreement with experiment.
\end{abstract}

PACS number(s): $34.80 . \mathrm{Dp}, 34.80 . \mathrm{Gs}, 34.80 . \mathrm{Kw}$

\section{INTRODUCTION}

Electron-impact ionization cross sections are widely used in applications such as the modeling of fusion plasmas in tokamaks, modeling of radiation effects for both materials and medical research, and aeronomy, as well as in basic research in astrophysics, atomic, molecular, and plasma physics. Although the method we present in this article-to be referred to as the binary-encounterdipole (BED) model-is equally applicable to ionization of atoms and molecules, we shall hereafter refer to the targets as "atoms" for brevity.

Ionization cross sections at all energies of incident particles and ejected electrons are needed to follow the history of an incident particle and its products for all ranges of energy transferred in individual collisions. Proper understanding of the role of ejected electrons is crucial because a large number of them, mostly slow electrons, are generated in the course of an energetic incident particle penetrating through matter. These electrons in turn interact with other targets until the electrons are thermalized.

Electron-atom collisions can be divided into two broad types: soft or distant collisions with large impact parameters and hard or close collisions with small impact parameters. The Mott theory [1], which describes the collision of two free electrons, accounts for hard collisions well but not soft collisions [2]. Bethe [3] has shown that soft collisions take place essentially through the dipole interaction between the incident particle and the target electron.

The symmetric form of the binary-encounter theory described by Vriens [4], which is meant for electron-impact ionization, augments the Mott formula by assigning a velocity or momentum distribution to a target electron instead of a wave function, but still lacks the dipole contribution and hence leads to an incorrect cross section when the dipole interaction dominates at high incident energies. In contrast, contributions from hard collisions dominate at lower incident energies.

There have been many attempts to combine the dipole contribution with either the Rutherford or Mott cross section to derive the "correct" ionization cross sections $[5,6]$, but these attempts have all had only limited success because they failed to find the correct mixing ratio between the expressions for the soft and hard collisions. In addition, since the scattered and ejected electrons are indistinguishable after an ionizing collision, electron exchange effect must be included, as is the case for the Mott and Vriens formulas.

Other approaches to represent ionization cross sections in compact, analytic forms [2,7-10] require parameters that must be fitted to some theoretical or experimental ionization cross sections.

The BED model uses the relation between the asymptotic (i.e., high incident energy) cross sections for ionization and for stopping (defined later) to deduce the mixing ratios not only between the soft and hard collisions but also for the electron exchange term. The BED 
model, however, does not depend on any empirical or fitted parameters, though accurate experimental or theoretical data on binding energies and photoionization cross sections are needed.

An important ingredient of the BED model is the differential dipole oscillator strength for ionization, which can be derived from either theoretical or experimental photoionization cross sections. The BED model constructs ionization cross sections subshell by subshell, and in principle can be used to construct ionization cross sections for any target atom or molecule as long as corresponding differential oscillator strengths for ionization are known. When high accuracy $(10 \%$ or better) is not required, differential oscillator strengths calculated from Hartree-Fock or similar wave functions are sufficient.

We also have included a simpler version of the BED model, to be referred to as the binary-encounter-Bethe (BEB) model, which may be used when the required differential oscillator strengths are not available.

We present a brief outline of the underlying theory in Sec. II, our BED model in Sec. III, the BEB model in Sec. IV, discussions of the total ionization cross section in Sec. V, modification of the theory for ion targets in Sec. VI, and comparisons of our results with experiments in Sec. VII. Our conclusions are presented in Sec. VIII. Often applications of ionization cross sections require total ionization cross sections only. In such cases, the total ionization cross sections calculated from the present theory can be recast into a simpler function of the incident electron energy. This simpler form is discussed in the Appendix.

\section{RUTHERFORD, MOTT, AND BINARY-ENCOUNTER CROSS SECTIONS}

\section{A. Rutherford cross section}

The collision of a particle with charge $Z_{1} e$ with a free electron at rest is described by the Rutherford cross section [11]:

$$
\frac{d \sigma(W, T)}{d W}=\frac{4 \pi a_{0}^{2} Z_{1}^{2} R^{2}}{T} \frac{1}{W^{2}}
$$

where $W$ is the kinetic energy of the ejected electron, $a_{0}$ is the Bohr radius $\left(=5.29 \times 10^{-11} \mathrm{~m}\right), R$ is the Rydberg energy $(=13.6 \mathrm{eV})$, and $T$ is the reduced kinetic energy defined by

$$
T=m v^{2} / 2 \text {, }
$$

with the relative speed $v$ and the electron mass $m$ regardless of the actual mass of the projectile. For incident electrons, $T$ is the nonrelativistic kinetic energy.

\section{B. Mott cross section}

Mott generalized the Rutherford cross section for the collision of two electrons $[1,11]$ to take account of exchange: $\frac{d \sigma(W, T)}{d W}=\frac{4 \pi a_{0}^{2} R^{2}}{T}\left[\frac{1}{W^{2}}-\frac{1}{W(T-W)}+\frac{1}{(T-W)^{2}}\right]$

In this nonrelativistic formula, $T$ is the kinetic energy of the incident electron, and $T-W$ is the kinetic energy of the scattered electron. The Mott cross section above is given in the form of a singly differential cross section (SDCS), or the energy distribution of the ejected electron.

Because the scattered and ejected electrons are indistinguishable, it is customary to call the faster one of the two (after a collision) the primary electron and the slower one the secondary electron. The first term in the square brackets of Eq. (3) is the direct collision term, the second term represents the interference between the direct and exchange collision terms, and the third term is the exchange collision term. Note that the Mott cross section is symmetric in the kinetic energies of the secondary electron, $W$, and the primary electron, $T-W$, as it should be. Both the Rutherford and the Mott cross sections, however, diverge when $W \rightarrow 0$ or when $W \rightarrow T$ in the case of electron-electron collision.

Of course, for a real atom, the cross section for ejecting an electron with $W=0$ is finite, and $W$ cannot be equal to $T$ because the binding energy must be overcome for a bound electron to be ejected. With these restrictions in mind, one can slightly modify the Rutherford and Mott cross sections by replacing $W$ by the energy transfer

$$
E=W+B,
$$

where $B$ is the binding energy of the ejected electron. With this substitution, the modified Rutherford cross section for a subshell becomes

$$
\frac{d \sigma(W, T)}{d W}=\frac{d \sigma}{d E}=\frac{4 \pi a_{0}^{2} Z_{1}^{2} N}{T} \frac{R^{2}}{E^{2}},
$$

where we have included the number of bound electrons, $N$, in the subshell. Similarly, the modified Mott cross section for a subshell is, after replacing $W$ by $E$ and $T$ by $T+B$ in the square brackets of Eq. (3), given by

$$
\begin{aligned}
\frac{d \sigma(W, T)}{d W}=\frac{d \sigma}{d E}= & \frac{4 \pi a_{0}^{2} R^{2} N}{T}\left[\frac{1}{E^{2}}-\frac{1}{E(T-W)}\right. \\
& \left.+\frac{1}{(T-W)^{2}}\right]
\end{aligned}
$$

Note that, while the original Mott cross section, Eq. (3), is an exact solution for the collision of two free electrons, the modified Mott cross section, Eq. (6), is an approximation for a bound target electron. In fact, Eq. (6) becomes a good approximation for ejecting a fast electron only when $W \gg B$.

\section{Binary-encounter cross section}

An extension of the Mott cross section to describe the ionization of a bound electron is to assign a velocity or 
momentum distribution to the target electron to represent the orbital motion of the bound electron. Although one can regard the Mott cross section itself as a formula that describes a binary collision, i.e., a billiard-ball-like collision between two free electrons, it is more common in a binary-encounter theory to associate some kind of momentum or velocity distribution with the target particle. Such a momentum distribution is often derived from the wave function for the target electron.

The symmetric form (i.e., for the primary and secondary electrons) of the binary-encounter theory [4] differs from the Mott cross section in that it has an extra term and introduces the average kinetic energy $U$ :

$$
\begin{aligned}
\frac{d \sigma(E, T)}{d E}= & \frac{4 \pi a_{0}^{2} R^{2} N}{T+U+B}\left\{\frac{1}{E^{2}}-\frac{1}{E(T-W)}\right. \\
& \left.+\frac{1}{(T-W)^{2}}+\frac{4 U}{3}\left[\frac{1}{E^{3}}+\frac{1}{(T-W)^{3}}\right]\right\} .
\end{aligned}
$$

In Eq. (7),

$$
U \equiv\left\langle\vec{p}^{2}\right\rangle / 2 m
$$

where $\vec{p}$ is the momentum operator of the electrons in a subshell.

At this point, it is convenient to express cross sections in terms of energy variables in units of the binding energy $B$ of the electrons in a subshell:

$$
\begin{aligned}
t & =T / B \\
w & =W / B \\
u & =U / B \\
S & =4 \pi a_{0}^{2} N(R / B)^{2} .
\end{aligned}
$$

With these reduced variables, the modified Mott cross section becomes

$$
\begin{aligned}
& \frac{d \sigma(W, T)}{d W}= \frac{d \sigma(w, t)}{B d w} \\
&=\frac{S}{B t}\left[\frac{1}{(w+1)^{2}}-\frac{1}{(w+1)(t-w)}\right. \\
&\left.+\frac{1}{(t-w)^{2}}\right] .
\end{aligned}
$$

Similarly, the binary-encounter cross section, Eq. (7), can be rewritten as

$$
\begin{aligned}
\frac{d \sigma(W, T)}{d W}= & \frac{S}{B(t+u+1)}\left\{\frac{1}{(w+1)^{2}}+\frac{1}{(t-w)^{2}}\right. \\
& -\frac{1}{(w+1)(t-w)} \\
& \left.+\frac{4 u}{3}\left[\frac{1}{(w+1)^{3}}+\frac{1}{(t-w)^{3}}\right]\right\}
\end{aligned}
$$

\section{BINARY-ENCOUNTER-DIPOLE (BED) MODEL}

Both the Mott and the binary-encounter cross sections for electron-impact ionization can be recast as a series:

$$
\frac{d \sigma(W, T)}{d W}=\frac{S}{B} \sum_{n=1}^{3} F_{n}(t)\left[\frac{1}{(w+1)^{n}}+\frac{1}{(t-w)^{n}}\right]
$$

The term containing $w+1$ represents the secondary electrons ejected from the target during the collision while the term containing $t-w$ describes the scattered primary electrons which have lost energy. Both types of electrons are detected in experiment, and they are indistinguishable. As was mentioned earlier, the $n=1$ term in Eq. (15) represents interference between the primary and secondary electrons, and the $n=2$ term arises from close collisions. The $n=3$ term accounts for the broadening of the energy distribution due to the intrinsic momentum distribution of a bound electron being ionized.

The Mott cross section corresponds to the following choice of $F_{n}(t)$ :

$$
F_{1}=-\frac{F_{2}}{t+1}, \quad F_{2}=\frac{1}{t}, \quad F_{3}=0
$$

and the binary-encounter cross section is reproduced by choosing

$F_{1}=-\frac{F_{2}}{t+1}, \quad F_{2}=\frac{1}{t+u+1}, \quad F_{3}=\frac{4 u}{3(t+u+1)}$

The total ionization cross section $\sigma_{i}$, which is obtained by integrating the SDCS [Eq. (15)] from $w=0$ to $(t-1) / 2$, reduces to a simple expression

$$
\begin{aligned}
\sigma_{i} & =\int_{0}^{(t-1) / 2} \frac{d \sigma}{d w} d w \\
& =S\left[F_{1} \ln t+F_{2}\left(1-t^{-1}\right)+\frac{1}{2} F_{3}\left(1-t^{-2}\right)\right]
\end{aligned}
$$

One can see that Eq. (19) with Eq. (16) or (17) leads to the asymptotic $(t \gg 1)$ behavior $\sigma_{i} \rightarrow t^{-1}$, which does not agree with the predictions of the Bethe theory nor with experiment. An example of this failure is shown in Sec. VIID. A more realistic asymptotic $t$ dependence is predicted by the Bethe theory [3], viz., $t^{-1} \ln t$, which arises from the dipole interaction.

To correct this deficiency, we consider the asymptotic case first. In the asymptotic region $t \gg w$, and hence the $(t-w)^{-n}$ terms may be ignored, i.e.,

$$
\frac{d \sigma}{d w}=S \sum_{n=1}^{3} F_{n}(t) f_{n}(w)
$$

with

$$
f_{n}(w)=\frac{1}{(w+1)^{n}} \quad \text { for } n=1,2
$$

while $F_{n}(t)$ and $f_{3}(w)$ are to be determined.

We now introduce the stopping cross section for ion- 
ization $\sigma_{\text {sti }}$, which is defined by

$$
\sigma_{\mathrm{sti}} \equiv B \int_{0}^{(t-1) / 2}(w+1) \frac{d \sigma}{d w} d w
$$

The asymptotic limits of $\sigma_{i}$ and $\sigma_{\text {sti }}$ are obtained by substituting Eq. (20) into Eqs. (18) and (22):

$$
\sigma_{i}=S\left(F_{1} \ln t+F_{2}+F_{3} G\right)
$$

where

$$
G \equiv \int_{0}^{\infty} f_{3}(w) d w
$$

and

$$
\sigma_{\mathrm{sti}}=\frac{S B}{R}\left[(\ln 2) F_{1} t+F_{2} \ln t+F_{3} H\right]
$$

where

$$
H \equiv \int_{0}^{\infty}(w+1) f_{3}(w) d w
$$

The upper limits of the integration for $G$ and $H$ have been extended to $\infty$ in the anticipation that $f_{3}(w)$ diminishes rapidly enough as $w \rightarrow \infty$ such that the asymptotic part of $f_{3}(w)$ does not contribute to the asymptotic $t$ dependence in Eqs. (23) and (25). This requires that $f_{3}(w) \rightarrow w^{-m}$ with $m>2$ for $w \gg 1$.

The corresponding asymptotic cross sections derived by Bethe using the first Born approximation are $[3,12]$

$$
\sigma_{i}=\frac{S Q}{2} \frac{\ln t}{t}
$$

where

$$
Q=\frac{2 B M_{i}^{2}}{N R}, \quad M_{i}^{2}=\frac{R}{B} \int_{0}^{\infty} \frac{1}{w+1} \frac{d f(w)}{d w} d w
$$

and

$$
\sigma_{\mathrm{sti}}=\frac{2 S B}{R} \frac{\ln t}{t}
$$

On the right-hand side (RHS) of Eq. (29), we have used the asymptotic form of the Bethe cross section for $\sigma_{\text {st }}$, which (unlike $\sigma_{\text {sti }}$ ) includes excitations to both discrete and continuum states. We made this approximation for two reasons. The first is that the coefficient of the $t^{-1} \ln t$ term for ionization not only is difficult to calculate but will also be different from one atom to another. The second reason is that at high incident energy, ionizing collisions account for $80 \%$ or more of $\sigma_{\text {st }}$ [13], and hence using the asymptotic dependence of $\sigma_{\text {st }}$ on the RHS of Eq. (29) is a simple yet effective approximation.

Matching Eqs. (23) and (25) with Eqs. (27) and (29) leads to

$$
\begin{aligned}
F_{1} \ln t+F_{2}+F_{3} G & =\frac{Q}{2} \frac{\ln t}{t} \\
(\ln 2) F_{1} t+F_{2} \ln t+F_{3} H & =2 \frac{\ln t}{t}
\end{aligned}
$$

There is no function $F_{1}(t)$ that will yield a $t^{-1} \ln t$ dependence in both Eqs. (30) and (31). Hence, we choose $F_{1}$ to make the first term in both equations fall off faster than $t^{-1} \ln t$ so as to become asymptotically negligible in both equations. The simplest way to accomplish this is to choose $F_{1} \propto t^{-2}$. This is consistent with the $t$ dependence of $F_{1}$ in the Mott and the binary-encounter cross sections provided that we make $F_{1}$ negative, i.e., make the interference term negative. If we now choose $F_{2} \propto t^{-1}$ as in the Mott and the binary-encounter cross sections, the second term in Eq. (30) becomes negligible but the second term in Eq. (31) matches the $t^{-1} \ln t$ dependence in the remainder of that equation.

With these choices for $F_{1}$ and $F_{2}$, the third term dominates in the asymptotic equation for $\sigma_{i}$, Eq. (30), while the second and the third terms dominate in the asymptotic equation for $\sigma_{\text {sti }}$, Eq. (31).

The asymptotic expression for the SDCS in the Bethe theory [14] is

$$
\frac{d \sigma}{d w}=\frac{S \ln t}{N t} \frac{1}{w+1} \frac{d f(w)}{d w}
$$

where $d f(w) / d w$ is the differential oscillator strength. On the other hand, the asymptotic limit of Eq. (20) is

$$
\frac{d \sigma}{d w}=S F_{3}(t) f_{3}(w)
$$

From Eqs. (32) and (33), we get

$$
F_{3}(t) f_{3}(w)=\frac{\ln t}{N t} \frac{1}{w+1} \frac{d f}{d w}
$$

An obvious choice is to set

$$
F_{3}(t)=\frac{\ln t}{t} \quad \text { and } \quad f_{3}(w)=\frac{1}{N(w+1)} \frac{d f(w)}{d w} .
$$

From this choice of $f_{3}(w)$, the definition of $M_{i}^{2}$ [Eq. (28)], and Eq. (24), we get

$$
\begin{aligned}
G & =\frac{1}{N} \int_{0}^{\infty} \frac{1}{w+1} \frac{d f(w)}{d w} d w \\
& =\frac{B M_{i}^{2}}{R N}
\end{aligned}
$$

and from Eq. (26)

$$
H=\frac{1}{N} \int_{0}^{\infty} \frac{d f(w)}{d w} d w=\frac{N_{i}}{N},
$$

where

$$
N_{i} \equiv \int_{0}^{\infty} \frac{d f(w)}{d w} d w
$$

Earlier, we mentioned that $f_{3}(w)$ for $w \gg 1$ should diminish as $w^{-m}$ with $m>2$ so that the upper limits in Eqs. (24) and (26) can be extended to $\infty$. This requirement is satisfied by our choice of $f_{3}(w)$ since $d f / d w$ diminishes in the asymptotic region as $w^{-m}$ where $m \geq 3.5$ [15]. 
To find $F_{2}(t)$, let $F_{2}=a / t$. Then, from the second and third terms of Eq. (31),

$$
\frac{a \ln t}{t}+\frac{N_{i} \ln t}{N t}=\frac{2 \ln t}{t}
$$

which leads to

$$
a=2-\left(N_{i} / N\right)
$$

or

$$
F_{2}(t)=\frac{2-\left(N_{i} / N\right)}{t} \text { and } F_{3}(t)=\frac{\ln t}{t} \text { for } t \gg 1
$$

What we have done so far is basically to let the $F_{3} f_{3}$ term in Eq. (15) represent the dipole interaction. The Bethe theory normally is not used in a symmetric form, i.e., it does not have the exchange and interference terms as in the Mott cross section. At present, it is not clear what the appropriate symmetric form of the dipole interaction should be. We simply omit the exchange term $(t-w)^{-3}$ in Eq. (15) until this question is resolved in the future. Besides, the exchange interaction becomes negligible at high incident energies where the dipole interaction is important.

Finally, we combine our choice for $F_{n}$ and $f_{3}$ with the symmetric binary-encounter cross section, Eq. (17), and set

$$
F_{1}=-\frac{F_{2}}{t+1}, \quad F_{2}=\frac{2-\left(N_{i} / N\right)}{t+u+1}, \quad F_{3}=\frac{\ln t}{t+u+1} .
$$

With Eqs. (15) and $f_{3}(w)$ defined in Eq. (35), the SDCS of a subshell based on the BED model is given by

$$
\begin{aligned}
\frac{d \sigma(W, T)}{d W}= & \frac{S}{B(t+u+1)}\left\{\frac{\left(N_{i} / N\right)-2}{t+1}\left(\frac{1}{w+1}+\frac{1}{t-w}\right)\right. \\
& \left.+\left[2-\left(N_{i} / N\right)\right]\left[\frac{1}{(w+1)^{2}}+\frac{1}{(t-w)^{2}}\right]+\frac{\ln t}{N(w+1)} \frac{d f(w)}{d w}\right\}
\end{aligned}
$$

According to the binary-encounter theory, the extra terms in the denominators of Eqs. (43) - $u$ and $1-$ represent the acceleration of the incident electron due to the nuclear attraction. These extra terms are essential in producing reliable ionization cross sections for low incident energies.

Now we look at the threshold behavior of the SDCS, i.e., the dependence as $t \rightarrow 1$. Let $t=1+\Delta t$ with $\Delta t \ll 1$. Then, our choice of $F_{n}$ and $f_{n}(w)$ leads to [from Eq. (44)]

$$
\sigma_{i}(1+\Delta t)=\text { const } \times \Delta t
$$

Although the Wannier theory [16] predicts $\sigma_{i} \propto(\Delta t)^{1.127}$ when $\Delta t$ is extremely small, Eq. (45) represents a more macroscopic threshold behavior [17].

To use the BED model, values of $B, U, N$, and the differential oscillator strengths, $d f / d w$, are needed for each subshell of a target. Of these, $B$ and $N$ are readily available from the literature. The values of $N_{i}$ and $M_{i}^{2}$ can be calculated from $d f / d w$. The average kinetic energy $U$ needed in the BED model is strictly a theoretical quantity, but can easily be obtained from wave function codes such as those developed by Froese-Fischer [18]. For oneelectron atoms, the virial theorem can be used to deduce $U$ from the known binding energy, i.e., $U=B$. However, one should not assume the virial theorem to hold for each subshell in atoms and molecules with complicated electronic structures. The virial theorem holds only between the total potential energy and the total kinetic energy. Binding and kinetic energies for subshells of many atoms and molecules are listed in Ref. [19]. Values of $U$ for $\mathrm{H}$, $\mathrm{He}, \mathrm{H}_{2}$, and $\mathrm{Ne}$ are included in Table $\mathrm{I}$.

\begin{tabular}{|c|c|c|c|c|c|c|c|}
\hline \multirow[b]{2}{*}{ Coeff. } & \multirow{2}{*}{$\begin{array}{l}\mathrm{H} \\
1 s\end{array}$} & \multirow{2}{*}{$\begin{array}{c}\mathrm{He} \\
1 s\end{array}$} & \multirow{2}{*}{$\begin{array}{c}\mathrm{H}_{2} \\
1 \sigma_{g}\end{array}$} & \multicolumn{4}{|c|}{$\mathrm{Ne}$} \\
\hline & & & & $2 p, \mathrm{I}^{\mathrm{B}}$ & $2 p, \mathrm{II}^{\mathbf{a}}$ & $2 s$ & $1 s$ \\
\hline$a$ & & & & 4.8791 & & & \\
\hline$b$ & $-2.2473[-2]$ & & & -2.8820 & -5.8514 & 1.7769 & \\
\hline$c$ & 1.1775 & $1.2178[1]$ & 1.1262 & $-7.4711[-1]$ & $3.2930[2]$ & 2.8135 & 5.2475 \\
\hline$d$ & $-4.6264[-1]$ & $-2.9585[1]$ & 6.3982 & & $-1.6788[3]$ & $-3.1510[1]$ & -2.8121 \\
\hline$e$ & $8.9064[-2]$ & $3.1251[1]$ & -7.8055 & & $3.2985[3]$ & $6.3469[1]$ & \\
\hline$f$ & & $-1.2175[1]$ & 2.1440 & & $-2.3250[3]$ & $-5.2528[1]$ & \\
\hline$g$ & & & & & & $1.5982[1]$ & \\
\hline $\bar{B}$ & $1.36057[1]$ & $2.459[1]$ & $1.543[1]$ & $2.160[1]$ & $2.160[1]$ & $4.847[1]$ & $8.669[2]$ \\
\hline$U$ & $1.36057[1]$ & $3.951[1]$ & $2.568[1]$ & $1.1602[2]$ & $1.1602[2]$ & $1.4188[2]$ & $1.2591[3]$ \\
\hline$M_{i}^{2}$ & 0.2834 & 0.489 & 0.680 & & $1.552^{\mathrm{b}}$ & $4.800[-2]$ & $1.642[-2]$ \\
\hline$N_{i}^{i}$ & 0.4343 & 1.605 & 1.173 & & $6.963^{b}$ & $7.056[-1]$ & 1.686 \\
\hline
\end{tabular}

Differential oscillator strengths are harder to get, although total and partial values of $d f / d w$ for many atoms and molecules may be found in the literature. Good

TABLE I. Power-series fit to differential oscillator strengths of $\mathrm{H}, \mathrm{He}, \mathrm{H}_{2}$, and $\mathrm{Ne}$. $\left[d f / d(E / B)=a y+b y^{2}+c y^{3}\right.$ $+d y^{4}+e y^{5}+f y^{6}+g y^{7}$, where $y=B / E, E=$ photon energy.] Numbers in square brackets are powers of ten, $B$ (in $\mathrm{eV}$ ) is the binding energy, and $U$ (in $\mathrm{eV}$ ) is the average kinetic energy.

${ }^{a} \mathrm{Ne}(2 p, \mathrm{I})$ covers photon energies from the $2 p$ ionization threshold $(21.60 \mathrm{eV})$ to the $2 s$ ionization threshold (48.47 eV), and $\mathrm{Ne}(2 p, \mathrm{II})$ covers photon energies from $48.47 \mathrm{eV}$ and above.

${ }^{\mathrm{b}}$ This number is the sum of $2 p, \mathrm{I}$ and $2 p, \mathrm{II}$ contributions. 
sources are the book by Berkowitz [20] and the review article by Gallagher et al. [21], from which one can find original references. For convenience, we have listed values of $N_{i}$ and $M_{i}^{2}$ for $\mathrm{H}, \mathrm{He}, \mathrm{Ne}$, and $\mathrm{H}_{2}$ in Table I and also presented values of $d f / d(E / B)$, where $E$ is the photon energy, in the form of simple polynomials of $B / E$. The differential oscillator strength for $\mathrm{H}$ is a fit to the exact theoretical values, and the others are fits to the experimental values compiled and recommended by Berkowitz [20]. We emphasize that we have used experimental $d f / d w$ when they are more reliable than calculated ones, particularly near the threshold. Although any form of theoretical $d f / d w$ can be used, analytic fits are certainly more convenient to use than numerical tables or graphs.

\section{BINARY-ENCOUNTER-BETHE (BEB) MODEL}

Although the BED model is substantially simpler to use than most $a b$ initio theories for electron-impact ionization, it is often difficult to get the differential oscillator strengths, particularly subshell by subshell. For such cases, we offer a simplified version, to be referred to as the binary-encounter-Bethe (BEB) model, in which a simple function is used for the $f_{3}(w)$ in Eq. (20).

Sometimes, the values of $N_{i}$ and/or $M_{i}^{2}$ for an atom are known but not the details of the corresponding $d f / d w$, because $N_{i}$ and $M_{i}^{2}$ can be determined from appropriate sum rules without $d f / d w$ if accurate oscillator strengths for discrete excitations are known [22]. In general, there is no simple relationship between $N_{i}$ and $M_{i}^{2}$ and hence knowing $N_{i}$ does not automatically lead to the value of $M_{i}^{2}$ unless the details of $d f / d w$ are also known.

In the BEB model, we assume a simple form of $d f / d w$, which can be integrated to obtain $N_{i}$ and $M_{i}^{2}$. However, these quantities can be replaced by better values if they are known. The BEB model even offers help when nothing is known about $N_{i}, M_{i}^{2}$, and $d f / d w$, though the reliability of resulting cross sections may suffer in this case.

As will be shown in Sec. VII, differential oscillator strengths for $\mathrm{H}, \mathrm{He}$, and $\mathrm{H}_{2}$ have simple shapes (except for resonances which are not important for our purpose) which can be represented by inverse powers of $w+1$, starting from $(w+1)^{-2}$, that is,

$$
\frac{d f}{d w}=\frac{b}{(w+1)^{2}}+\cdots
$$

where $b$ is a constant. If we retain only the first term, then

$$
N_{i}=b \int_{0}^{\infty} \frac{d w}{(w+1)^{2}}=b
$$

and

$$
M_{i}^{2}=\frac{R b}{B} \int_{0}^{\infty} \frac{d w}{(w+1)^{3}}=\frac{R b}{2 B}
$$

By combining Eqs. (28), (47), and (48), we get

$$
M_{i}^{2}=R N_{i} / 2 B \text { and } Q=N_{i} / N
$$

Note that the specific relationship between $N_{i}$ and $M_{i}^{2}$ (and $Q$ ) above is a result of choosing $d f / d w$ to have the special form in Eq. (46). Equations (49) are not expected to hold for all subshells in targets with complicated shell structures.

After substituting Eqs. (46) (first term only) and (47) into Eq. (35), we have

$$
f_{3}(w)=Q /(w+1)^{3},
$$

which puts us back to the form of the binary-encounter cross section, Eq. (14), except that $4 u / 3$ is now replaced by $Q$. This similarity between Eq. (50) and the binaryencounter theory is probably the reason for the apparent success in the early days of the binary-encounter and other related classical theories (e.g., by Gryzinski [23]) on these targets with simple shell structures.

Since $f_{3}$ will always be used with $F_{3}$ as a product [see Eq. (34)], one can transfer $Q$ in Eq. (50) to $F_{3}$ to simplify our notation for $f_{3}$ :

$$
F_{3}(t)=\frac{Q \ln t}{t}, \quad \text { and } \quad f_{3}(w)=1 /(w+1)^{3} \text {. }
$$

With Eqs. (15), (43), (49), and (51), the SDCS in the $\mathrm{BEB}$ model is given by

$$
\frac{d \sigma}{d w}=S \sum_{n=1}^{3} F_{n}(t)\left[f_{n}(w)+f_{n}(t-w)\right]
$$

where

$$
f_{n}(w)=(w+1)^{-n}, \quad f_{n}(t-w)=(t-w)^{-n},
$$

and

$$
F_{1}=-\frac{F_{2}}{t+1}, \quad F_{2}=\frac{2-Q}{t+u+1}, \quad F_{3}=\frac{Q \ln t}{t+u+1}
$$

Equations (54) are most useful to estimate the total ionization cross section (TICS) when only the value of $M_{i}^{2}$ (and thus $Q=2 B M_{i}^{2} / N R$ ) is known. As a further approximation if $M_{i}^{2}$ is not available, we can set $Q=$ 1. This is a useful approximation that will still lead to ionization cross sections of correct orders of magnitude when nothing is known about differential or total dipole oscillator strengths.

\section{TOTAL CROSS SECTION FOR IONIZATION BY ELECTRON IMPACT}

Using Eqs. (15) and (43) with the $F_{n}$ and $f_{n}$ from the BED model, Eq. (18) reduces to a simple expression for $\sigma_{i}(t)$ for all $t$ :

$$
\begin{aligned}
\sigma_{i}(t)= & \frac{S}{t+u+1}[D(t) \ln t \\
& \left.+\left(2-\frac{N_{i}}{N}\right)\left(\frac{t-1}{t}-\frac{\ln t}{t+1}\right)\right]
\end{aligned}
$$


where

$$
D(t) \equiv N^{-1} \int_{0}^{(t-1) / 2} \frac{1}{w+1} \frac{d f(w)}{d w} d w
$$

and $N_{i}$ is defined by Eq. (39).

Note that the above cross section is for a given subshell, and these cross sections must be summed over all subshells that contribute to ionization. In practice, only the valence shell and a few subshells below it will make significant contributions to $\sigma_{i}$.

Equation (55) has the same asymptotic form as the Bethe theory [Eq. (27)] since from Eqs. (28) and (56) $D(\infty)=(B / R N) M_{i}^{2}$. The advantages of the BED formula for $\sigma_{i}$ over the Bethe theory-or other variations of the Bethe theory - are that the BED formula (a) is designed to approach the low $t$ region with an appropriate threshold behavior, (b) includes the interference term between the direct and exchange interactions, which manifests itself as the $\ln t /(t+1)$ term, and (c) leads to a reduction in the dipole contribution $D(t)$ as $t$ decreases. In Fig. 1, we compare the $\sigma_{i}$ for $\mathrm{H}$ deduced from the BED model with other theories as well as the experimental $\sigma_{i}$ measured by Shah et al. [24]. Note that the BED model does not contain any empirical or adjustable parameters. The TICS's based on the BED model for some simple atoms also agree well ( $\pm 10 \%$ or better in most cases) with available accurate measurements [see Sec. VII].

The TICS based on the BEB model is given by

$$
\begin{aligned}
\sigma_{i}(t)= & \frac{S}{t+u+1}\left\{\frac{1}{2} Q\left(1-\frac{1}{t^{2}}\right) \ln t\right. \\
& \left.+(2-Q)\left[\left(1-\frac{1}{t}\right)-\frac{\ln t}{t+1}\right]\right\},
\end{aligned}
$$

where $Q$ is defined by Eq. (28).

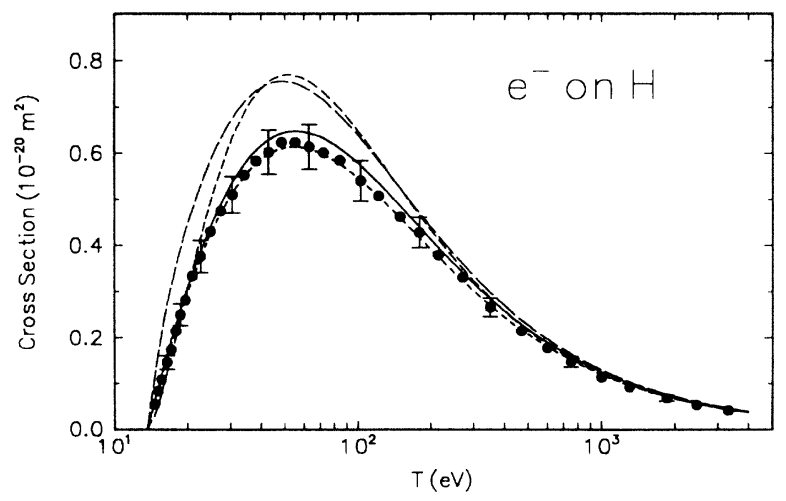

FIG. 1. Total cross section for ionization of $\mathrm{H}$ by electron impact. The abscissa is the incident electron energy $T$ in $\mathrm{eV}$. Filled circles, experimental data by Shah et al. [24]; solid line, BED cross section; short-dashed line, BEB cross section; medium-dashed line, Gryzinski's classical cross section [23]; long-dashed line, distorted-wave Born cross section with electron exchange correction by Younger [34].

\section{MODIFICATION FOR ELECTRON-ION COLLISIONS}

We chose our $F_{n}(t)$ to have $t+u+1$ in the denominator while the Mott and Bethe cross sections have only $t$. Our choice originated from the symmetric form of the binaryencounter theory [25] in which the incident electron was assumed to gain a kinetic energy of $U+B$ before the collision to make it equivalent to the target electron, which has a potential energy of $-|U+B|$. This denominator, $t+u+1$, reduces the cross section, which is the desired effect since most collision theories overestimate ionization cross sections near the peak. As is shown later, much of the success of our model for neutral targets can be attributed to this denominator.

The symmetric binary-encounter theory and the BED model do not distinguish between collisions with neutral atoms and those with ions, as far as the incident electron is concerned, although the target electron description differs through $U, B$, and $d f / d w$. The correct theory should, however, also alter the description of the incident electron since the long-range Coulomb force between the incident electron and a target ion should distort the wave function of the incident electron for its entire path, while such distortion should occur only in the vicinity of a neutral target. The Coulomb-Born and the distorted-wave Born approximations are examples in which such distortions are included. Qualitatively, the charge density of the incident electron is attracted toward the target ion, thus increasing the overlap between the charge densities of the incident and target electrons. This results in increased cross sections.

Indeed, we found the BED cross sections with $t+u+1$ in the denominator for the ionization of $\mathrm{He}^{+}$and $\mathrm{Li}^{2+}$ are too low compared to reliable experiments [26-29] as is shown later. Instead, we found that replacing $t+u+1$ in Eqs. (43) and (54) with $t+1$ resulted in better agreements with experiment. At this point, we cannot prove that $t+1$ is the correct denominator for ions. Furthermore, a preliminary application of the BED model to $\mathrm{Li}^{+}$ slightly favors the use of $t+u+1$ in the denominator. Hence we tentatively propose to use, for target ions with open-shell valence electrons,

$$
F_{1}=-\frac{F_{2}}{t+1}, \quad F_{2}=\frac{2-N_{i} / N}{t+1}, \quad F_{3}=\frac{\ln t}{t+1}
$$

for the BED model, and

$$
F_{1}=-\frac{F_{2}}{t+1}, \quad F_{2}=\frac{2-Q}{t+1}, \quad F_{3}=\frac{Q \ln t}{t+1}
$$

for the BEB model. We recommend to use $t+u+1$ in the denominator for ions with closed-shell configurations.

Actually, the differential dipole oscillator strength in units of appropriate binding energies, $d f / d(E / B)$, for one-electron ions is independent of the nuclear charge $Z$. The coefficients for the hydrogen atom in Table I may also be used for this universal $d f / d(E / B)$ in powers of $B / E$. This is a fit to the exact theoretical expression for the photoionization of a $1 s$ electron in a hydrogenic ion. 
The data in Table I can be used to calculate ionization cross sections using Eqs. (58).

For all one-electron ions [22], $M_{i}^{2}=0.2834 / Z^{2}$ and hence $Q=0.5668$. With these results, we have a simple, universal formula for the ionization of one-electron ions based on the BEB model:

$$
F_{1}=-\frac{1.4332}{(t+1)^{2}}, \quad F_{2}=\frac{1.4332}{t+1}, \quad F_{3}=\frac{0.5668 \ln t}{t+1}
$$

$$
\text { with } t=T / Z^{2} R \text {. }
$$

Equations (60) should be used with $f_{n}$ defined by Eqs. (53), and the matching BEB total ionization cross section for a one-electron atom is given by

$\sigma_{i}=\frac{4 \pi a_{0}^{2}}{Z^{4}}\left[F_{1}(t) \ln t+F_{2}(t)\left(1-t^{-1}\right)+\frac{1}{2} F_{3}(t)\left(1-t^{-2}\right)\right]$,

$$
\text { with } t=T / Z^{2} R \text {. }
$$

\section{COMPARISONS WITH EXPERIMENTS}

Before we compare the BED and BEB cross sections with available experimental data, we discuss a simple and powerful way to graphically compare theoretical and experimental results first proposed by Platzman to graphically represent SDCS's $[2,30]$. In his method, which we shall refer to as the Platzman plot, the ratio $Y$ of $d \sigma(W, T) / d W$ to the Rutherford cross section, Eq. (5) with $N$ and $Z_{1}$ equal to 1 , is plotted as a function of the inverse energy transfer $R / E$ :

$$
Y=\frac{d \sigma(W, T)}{d W} \frac{T}{4 \pi a_{0}^{2}} \frac{E^{2}}{R^{2}},
$$

where $d \sigma / d W$ can either be experimental or theoretical. If the target is a multishell atom, the lowest binding energy is used to define $E$ in Eq. (62). Since $d \sigma(W, T) / d W=d \sigma(E, T) / d E$,

$$
\int Y d\left(\frac{R}{E}\right)=\frac{T}{4 \pi a_{0}^{2} R} \int \frac{d \sigma(W)}{d W} d W=\frac{T}{4 \pi a_{0}^{2} R} \sigma_{i}
$$

In other words, the area under the Platzman plot is proportional to the TICS. This fact can be used to normalize $d \sigma / d W$ because $\sigma_{i}$ is often known with a better accuracy than $d \sigma / d W$ from independent measurements.

Similarly to the physical interpretation of the dipole oscillator strength, $Y$ can be interpreted as the effective number of target electrons participating in an ionizing collision. In this way, we expect that the value of $Y$ will approach the number of valence electrons as $W \gg B$ provided that the valence shell contributions dominate the TICS as is the case in most atoms and molecules. For slow secondary electrons, the shape of the Platzman plot is expected to follow the shape of the differential oscillator strengths (multiplied by the energy transfer) "scaled" by some function of $t$ and superposed on the contributions from close collisions, i.e., the Rutherford cross sec- tion. However, this scaling of the dipole contribution will be different from one atom to another. Because of the complexity of this scaling, one cannot simply add the dipole contribution to the Rutherford (or the Mott) cross section to "synthesize" SDCS. Nevertheless, the Platzman plot provides many useful clues to the reliability of experimental as well as theoretical $d \sigma / d W$, particularly when reliable values of differential oscillator strengths are known [31].

The success of the BED model is achieved by fixing this "scaling" between different components of the ionization cross section through Eqs. (43).

We present an example of the Platzman plot for the ionization of the hydrogen atom by $60-\mathrm{eV}$ electrons in Fig. 2. Experimental electron-impact data by Shyn [32] are presented in Fig. 2(a) and the corresponding (exact) differential oscillator strength of hydrogen in Fig. 2(b). We can see immediately that the shapes of the two curves are very similar, indicating that dipole contribu-

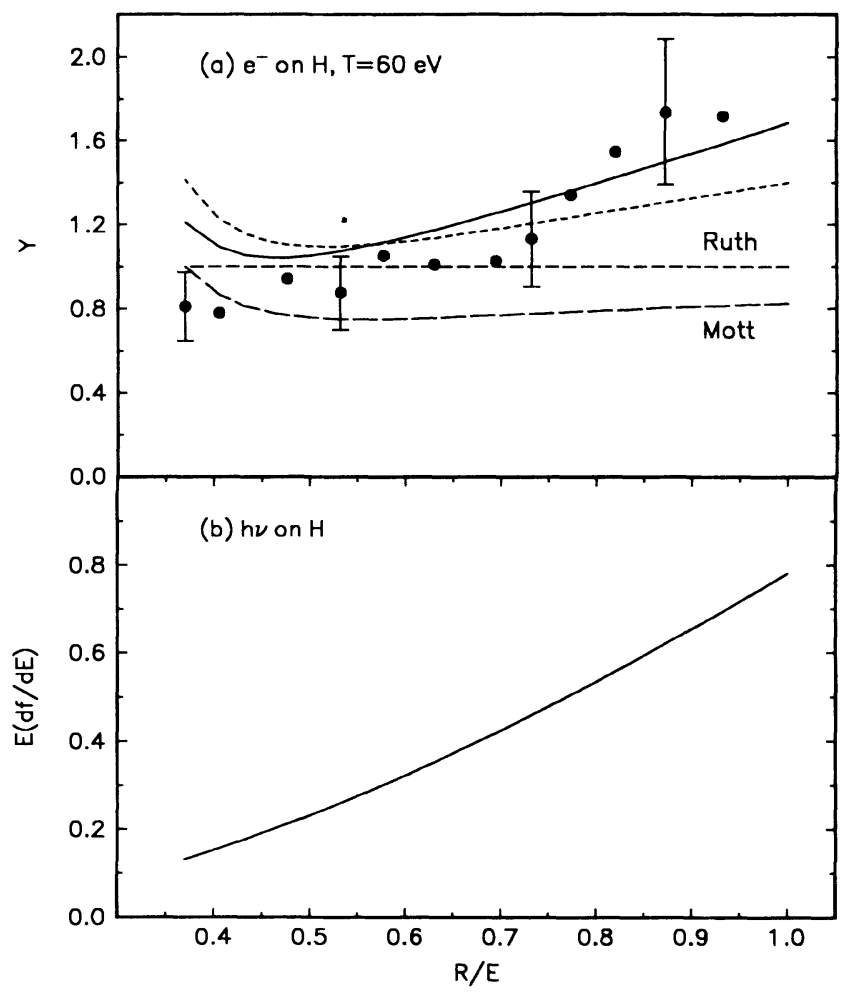

FIG. 2. Energy distribution of secondary electrons from $\mathrm{H}$ by electron impact. The abscissa is the inverse of the energy transferred to the target, $E$, in rydbergs. (a) Platzman plot of the SDCS at $T=60 \mathrm{eV}$. Filled circles, experimental data by Shyn [32]; solid line, BED cross section; short-dashed line, BEB cross section; medium-dashed line marked "Ruth," the Rutherford cross section; long-dashed line marked "Mott," Mott cross section. Shyn's data were renormalized so that the integrated cross section - which is proportional to the area under the data points between $R / E=1$ and 0.370 -matches the total ionization cross section at $T=60 \mathrm{eV}$ in Fig. 1. (b) Differential oscillator strength $d f / d E$ for ionization of $\mathrm{H}(1 s)$. The ordinate is the dipole function $E(d f / d E)$ that matches the dipole contribution in a Platzman plot. The energy transferred, $E$, is the actual photon energy. 
tions are prominent at the incident energy of $T=60 \mathrm{eV}$. The Mott cross section [Eq. (6)] is marked "Mott," while the Rutherford cross section [Eq. (5)] is marked "Ruth."

To match the accurate TICS measured by Shah et al. [24], however, the data by Shyn have been renormalized to the height shown in Fig. 2(a). The area under Shyn's data between the threshold $(R / E=1)$ and the upper limit of the secondary electron energy $(R / E=0.370)$ reproduces the electron-impact $\sigma_{i}$ measured by Shah et al. [24], $\sigma_{i}$ (expt.) $=6.16 \times 10^{-21} \mathrm{~m}^{2}$. The BED model leads to $\sigma_{i}(\mathrm{BED})=6.47 \times 10^{-21} \mathrm{~m}^{2}$, while the BEB result is $\sigma_{i}(\mathrm{BEB})=6.12 \times 10^{-21} \mathrm{~m}^{2}$ for $Q=0.5668$ and $6.60 \times 10^{-21} \mathrm{~m}^{2}$ for $Q=1$.

Note that it is very simple to extrapolate the electronimpact data for slow secondary electrons, $W<5 \mathrm{eV}$, using the shape of the dipole contribution in Fig. 2(b). This possibility is important because most experimental data on secondary electrons are either unavailable or unreliable at $W<5 \mathrm{eV}$.

Figure 2 clearly demonstrates the power of the Platzman plot in (a) checking the reliability of experimental cross sections, (b) normalizing the overall magnitude, and (c) extrapolating $d \sigma / d W$ to values of $W$ inaccessible to experiments, as long as the dipole contribution is discernible.

\section{A. Hydrogen atom}

In Fig. 1, the BEB cross section (short-dashed line) nearly coincides with the experimental $\sigma_{i}$ [24] while the $\mathrm{BED}$ cross section (solid line) is slightly higher than the experiment, though still within the experimental error limits. It is well known that the plane-wave Born cross section overestimates the peak cross section by about $65 \%$ [33]. The long-dashed line is the distorted-wave Born cross section that includes electron exchange correction by Younger [34]. The medium-dashed line is the cross section based on Gryzinski's classical theory [23].

Although the BEB cross section for the total ionization appears to agree better with the experiment than the BED cross section, Fig. 2(a) indicates that the shape of the experimental SDCS's by Shyn [32] is in better agreement with the shape of the BED model (solid line) than that of the BEB model (short-dashed line). The importance of using the correct $d f / d w$ will become more apparent when we compare the SDCS from the BED model with other experimental data (see Sec. VIIE).

At high incident energies, the dipole contribution to the SDCS - the difference between the solid line and the Mott cross section in Fig. 2(a)-increases while the Mott cross section remains almost constant, making the overall shape of the Platzman plot strongly resemble the shape of the dipole function in Fig. 2(b).

\section{B. One-electron ions}

In the nonrelativistic form, the differential oscillator strengths of hydrogenic ions scale as a function of $Z^{2}$, and hence it is simple to extend the BED cross section for the hydrogen atom to one-electron ions as outlined in Sec. VI.

Experimental TICS's for $\mathrm{He}^{+}$and $\mathrm{Li}^{2+}$ are available [26-29]. In Fig. 3, we compare our BED cross sections for total ionization using the $t+1$ denominator (solid line) and those using the $t+u+1$ denominator (mediumdashed line) with the experiment [26] as well as with Younger's distorted-wave Born cross section (dot-dashed line) [34] and Gryzinski's classical cross section (longdashed line) [23]. While both BED and BEB cross sections with the $t+u+1$ denominator are lower than the experimental data near the peak, the BED cross section with the $t+1$ denominator is in good agreement, as we have discussed in Sec. VI. Gryzinski's cross section is remarkably close to our BED cross section, but this is an accidental agreement, in view of the disagreement seen in the case of $\mathrm{H}$ and $\mathrm{H}_{2}$, for instance. The cross sections for the ionization of $\mathrm{He}^{+}$measured by Defrance et al. [27] and those by Achenbach et al. [28] are in good agreement with those by Peart et al. [26] and hence with our BED model with the $t+1$ denominator.

As is shown in Fig. 4, the BED cross section with the $t+1$ denominator (solid line) is in good agreement with the experimental data by Tinschert et al. [29]. The BED cross section with the $t+u+1$ denominator (long-dashed line) is too small, while Gryzinski's classical cross section (medium-dashed line) [23] merges with experiment only beyond the peak.

\section{Helium atom}

In Fig. 5, we compare our BED cross section (with the $t+u+1$ denominator for a neutral target) for the ionization of $\mathrm{He}$ with experimental data by Shah et al. (filled circles) [35] and those by Montague et al. (filled triangles) [36].

Again, we see excellent agreement between the BED cross section and the experimental data. The distorted-

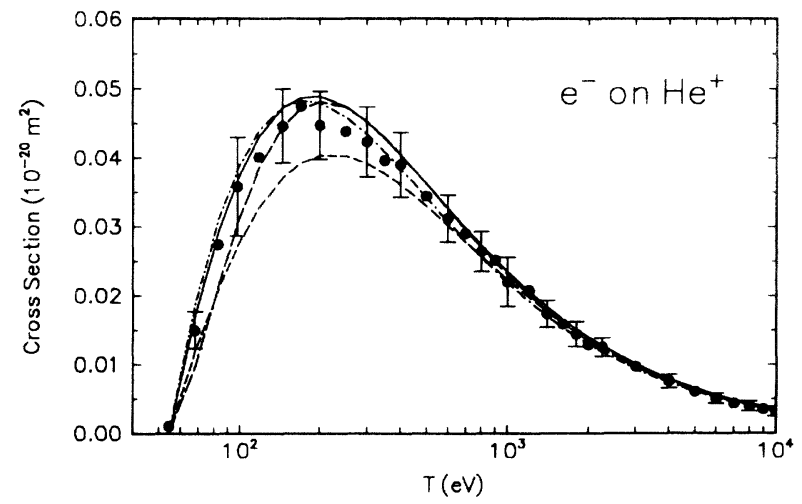

FIG. 3. Total cross section for ionization of $\mathrm{He}^{+}$by electron impact. Filled circles, experimental data by Peart et al. [26]; solid line, BED cross section with the $t+1$ denominator; medium-dashed line, the same with the $t+u+1$ denominator (see Sec. VI); long-dashed line, Gryzinski's classical cross section [23]; dot-dashed line, Younger's distorted-wave Born cross section [34]. 


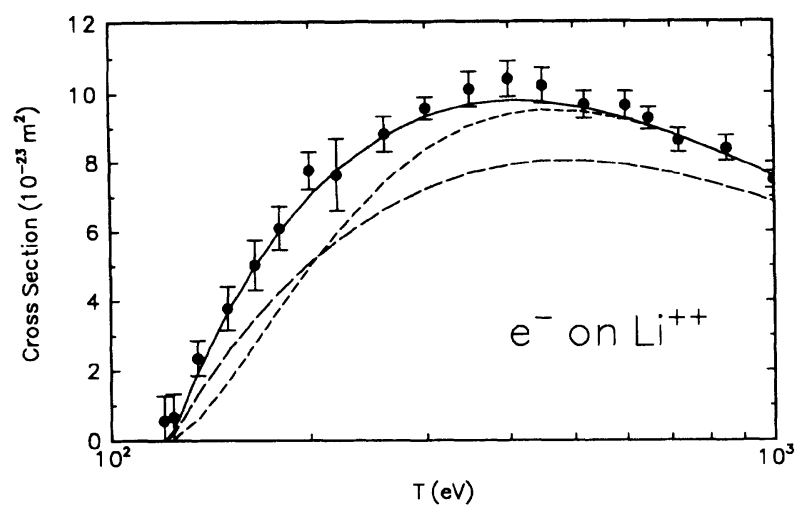

FIG. 4. Total cross section for ionization of $\mathrm{Li}^{2+}$ by electron impact. Filled circles, experimental data by Tinschert et al. [29]; solid line, BED cross section with the $t+1$ denominator; long-dashed line, the same with the $t+u+1$ denominator (see Sec. VI); medium-dashed line, Gryzinski's classical cross section [23].

wave Born cross section (medium-dashed line) by Younger [34] overestimates the peak cross section.

A Platzman plot of the SDCS of He by $500-\mathrm{eV}$ electrons is presented in Fig. 6. The solid line is our BED cross section, the filled circles are experimental data by Opal et al. [37], and the filled triangles are those by Goruganthu and Bonham [38]. One can see from the areas in the Platzman plot that the data by Opal et al. lead to too large a total cross section, while the normalization of the Goruganthu-Bonham data seems to be correct. The advantage of a Platzman plot is amply demonstrated by the small peak at $R / E \sim 0.23$, which represents the autoionization of the doubly excited $2 s 2 p$ state. The overall shape of the Platzman plot comes from that of the dipole function $E(d f / d E)$ for $\mathrm{He}$, which is similar in shape to that shown for $\mathrm{H}$ in Fig. 2(b), though the magnitude and abscissa are very different from those for $\mathrm{H}$.

The BED model is too simple to account for sharp autoionization peaks and resonances, although we could

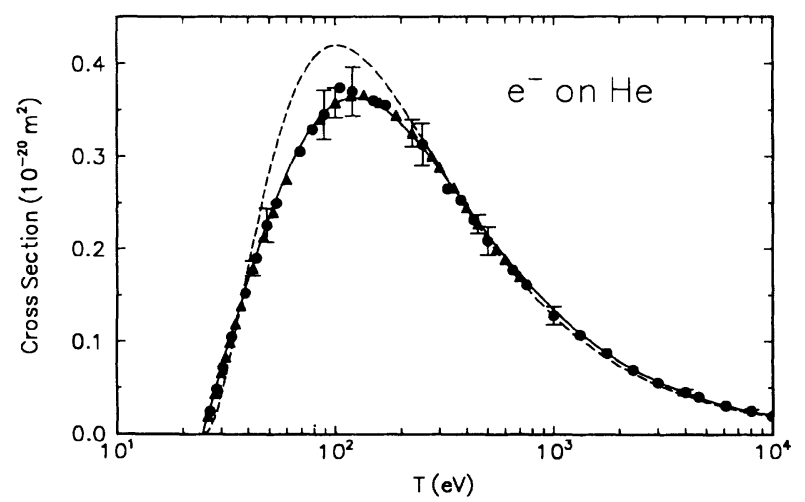

FIG. 5. Total cross section for ionization of He by electron impact. Filled circles, experimental data by Shah et al. [35]; filled triangles, data by Montague et al. [36]; solid line, BED cross section (with the $t+u+1$ denominator for a neutral target); medium-dashed line, Younger's distorted-wave Born cross section [34].

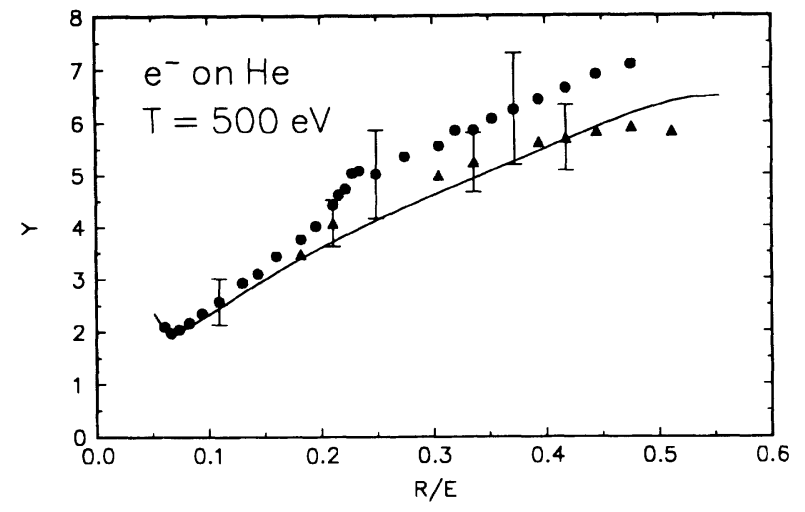

FIG. 6. Energy distribution of secondary electrons from He at $T=500 \mathrm{eV}$. This is a Platzman plot of SDCS of He. Solid line, BED cross section; filled circles, experimental data by Opal et al. [37]; filled triangles, data by Goruganthu and Bonham [38]. The small peak at $R / E \sim 0.23$ arises from the autoionization of the doubly excited state $2 s 2 p$.

have indicated their presence by including such details seen in photoionization experiments in the dipole function $E(d f / d E)$ we have used. The shape and height of such resonances in SDCS's, however, would have been incorrect because the BED model does not account for the intricate interference between the doubly excited states and the background continuum.

\section{Hydrogen molecule}

In Fig. 7, we compare our BED cross section (solid line) for the ionization of $\mathrm{H}_{2}$ with electron-impact experimental data by Rapp and Englander-Golden [39]. Although the BED cross section is somewhat smaller than the experimental data before reaching the peak, agreement between our theory and experiment is still far better than any theoretical efforts made in the past. Gryzinski's classical cross section (long-dashed line) overesti-

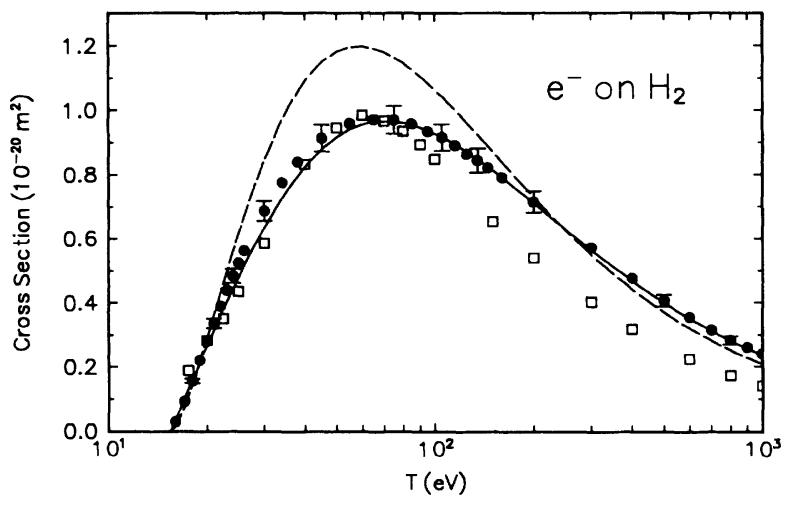

FIG. 7. Total cross section for ionization of $\mathrm{H}_{2}$ by electron impact. Filled circles, experimental data by Rapp and Englander-Golden [39]; open squares, calculations by Schultz et al. [40] based on the classical trajectory Monte Carlo (CTMC) method; solid line, BED cross section; long-dashed line, Gryzinski's classical cross section [23]. 
mates the peak cross section. The squares represent the classical trajectory Monte Carlo (CTMC) cross section calculated by Schultz et al. [40], which agrees well with experiment from threshold to the peak. The CTMC cross section begins to fall below the experimental data beyond the peak because CTMC cross sections lack the dipole contribution that increases at high incident energies.

A Platzman plot of SDCS at $T=100 \mathrm{eV}$ is presented in Fig. 8. Two sets of experimental data are available at this incident energy, those by Shyn et al. [41] (filled circles) and those by DuBois and Rudd [42] (filled triangles). Both sets of experimental data indicate a departure from the expected shape for slow secondary electrons, $W<10$ $\mathrm{eV}$. Since the lowest secondary electron energy measured by DuBois and Rudd is $W=4 \mathrm{eV}$, their data must be extrapolated to the threshold, $W=0 \mathrm{eV}$, before the corresponding integrated cross sections, $\sigma_{i}$ at $T=100$ $\mathrm{eV}$, can be determined. Judging from the area under the Platzman plot, the data by DuBois and Rudd would lead to $\sigma_{i}$ at $T=100 \mathrm{eV}$, significantly lower than that shown in Fig. 7. As shown in this example, the Platzman plot provides an effective guide to extrapolate SDCS's to the threshold, where experimental difficulties are the greatest.

\section{E. Neon atom}

The TICS is compared in Fig. 9, where the BED cross section (solid line) overestimates $\sigma_{i}$ at low incident energies, but agrees better with the measurement by Rapp and Englander-Golden [39] (filled circles) than the experiment by Wetzel et al. [43] (filled triangles).

In Fig. 10, the SDCS's at $T=500 \mathrm{eV}$ are compared. The solid line is the BED cross section, while the filled circles are the experimental data by Opal et al. [37], and the filled triangles are those by DuBois and Rudd [42]. The magnitudes of both sets of experimental data are too small to match the TICS's in Fig. 9. The data by Opal et al. resemble the expected shape-which comes

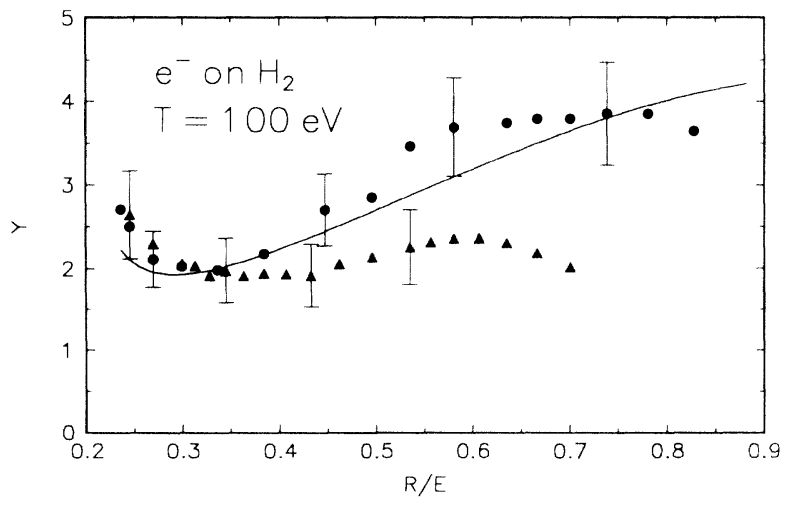

FIG. 8. Energy distribution of secondary electrons from $\mathrm{H}_{2}$ at $T=100 \mathrm{eV}$. This is a Platzman plot of SDCS of $\mathrm{H}_{2}$. Solid line, BED cross section; filled circles, experimental data by Shyn et al. [41]; filled triangles, data by DuBois and Rudd [42].

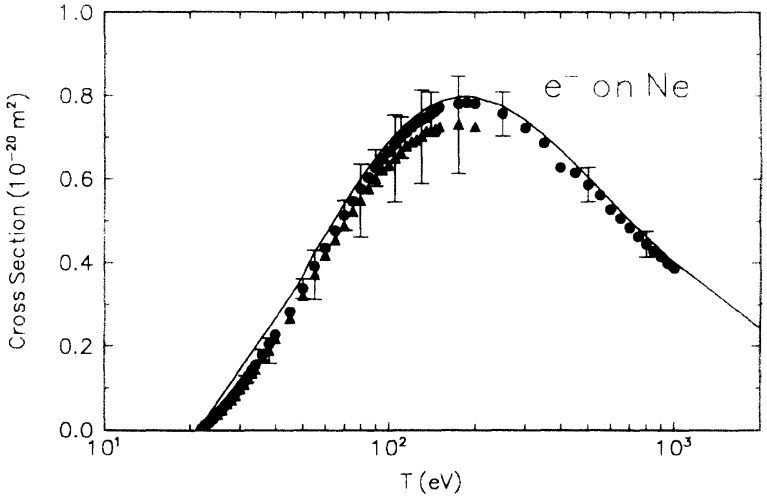

FIG. 9. Total cross section for ionization of $\mathrm{Ne}$ by electron impact. Solid line, BED cross section; filled circles, experimental data by Rapp and Englander-Golden [39]; filled triangles, data by Wetzel et al. [43].

from the shape of the dipole function $E(d f / d E)$ - while the data by DuBois and Rudd for slow secondaries are too low, as they themselves indicated [42].

The overall shape of the Platzman plot-with a peak at $R / E \sim 0.2$-reflects the shape of $E(d f / d E)$. Had we used the BEB model, the shape of the Platzman plot would have resembled that shown in Fig. 2.

\section{F. Water molecule}

In Figs. 11 and 12, it is seen that the BEB model may also be applied quite successfully to molecules with several molecular orbitals. Using $Q=1$ and values of $B$ and $U$ from Rudd et al. [19], the contributions to the SDCS's and TICS's from the five molecular orbitals were calculated and then summed. The total cross section is shown in Fig. 11 as a solid line. Considering the spread among the various experimental values, the agreement is satisfactory.

In Fig. 12, the SDCS's for $T=1500 \mathrm{eV}$ are com-

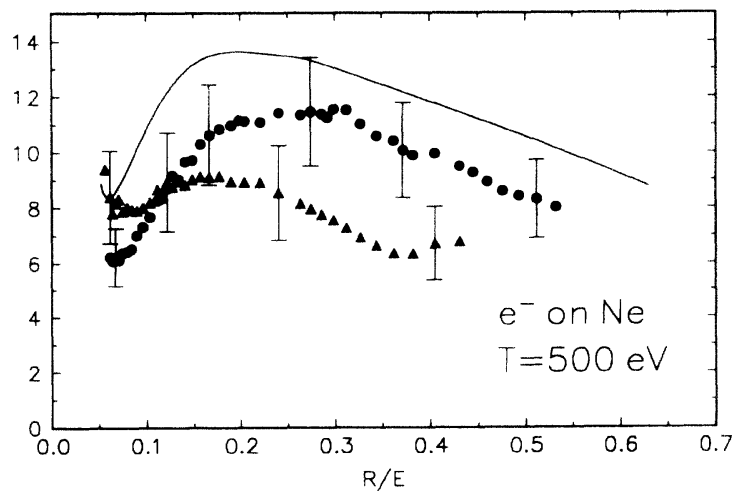

FIG. 10. Energy distribution of secondary electrons from Ne at $T=500 \mathrm{eV}$. This is a Platzman plot of SDCS of Ne. Solid line, BED cross section; filled circles, experimental data by Opal et al. [37]; filled triangles, data by DuBois and Rudd [42]. 


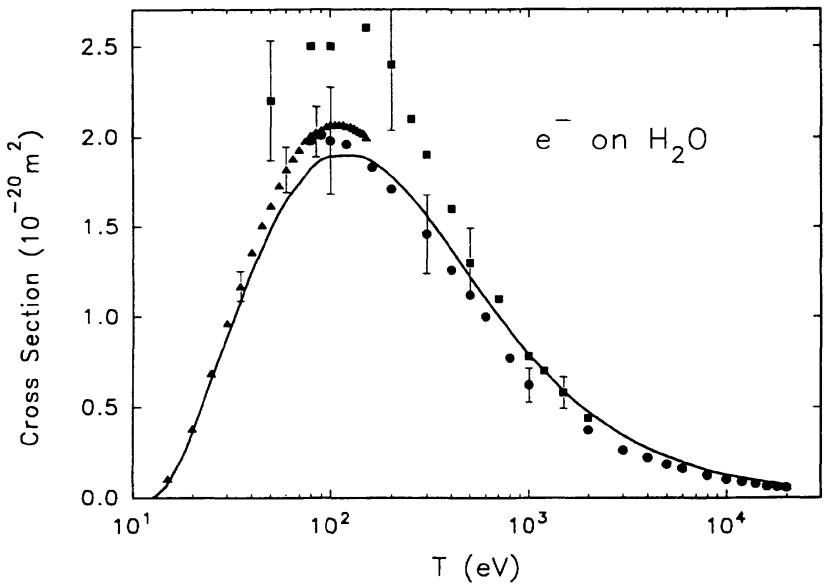

FIG. 11. Total cross section for ionization of water vapor by electron impact. Filled triangles, experimental data by Durić et al. [48]; filled squares, data by Bolorizadeh and Rudd [49]; filled circles, data by Schutten et al. [50]; solid line, BEB cross section.

pared with experimental data of Hollman et al. [44] and with calculations using the Miller model $[5,44]$. The peak near $500 \mathrm{eV}$ is from $K$ Auger transitions in oxygen and the sharp drop near $1000 \mathrm{eV}$ results from the fact that $1500-\mathrm{eV}$ incident electrons which have ionized $K$ electrons cannot have energies above that value. There are similar drops in the $1470-1490-\mathrm{eV}$ region due to the other molecular orbitals, but they are not resolved. Note that the ordinate in Fig. 12 is the ratio of $d \sigma / d W$ to the Mott cross section, unlike the Platzman plot. Also, the abscissa is the secondary electron energy itself, $W$, to

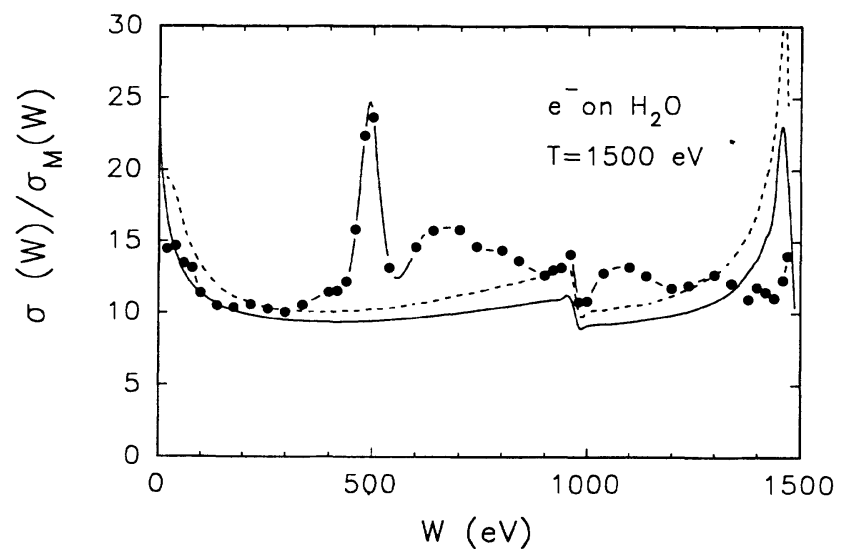

FIG. 12. Energy distribution of secondary electrons from water vapor at $T=1500 \mathrm{eV}$. The ordinate is the SDCS $[\sigma(W)]$ divided by the corresponding Mott cross section $\left[\sigma_{M}(W)\right.$, Eq. (6)]. Filled circles, experimental data by Hollman et al. [44]; dashed line, calculations using the Miller model [5,44]; solid line, calculations using the BEB model, Eqs. (52)-(54). Using the method described by Hollman et al. [44], the five highest-energy experimental points have been corrected for small-angle scattered primaries which were not detected. The bumps at about 650,1100 , and $1300 \mathrm{eV}$ in the experimental data are artifacts due to the coarseness of the angular mesh. present the details of the SDCS beyond the "operational" definition of the upper limit, $W_{\max }=(T-B) / 2$, of the secondary electron energy.

\section{CONCLUSIONS}

We have successfully combined the binary-encounter theory and the dipole interaction-referred to as the binary-encounter-dipole (BED) model - to provide a remarkably reliable method to predict singly differential and total ionization cross sections. Both types of cross sections have three basic components: the electron exchange term, the hard collision term, and the dipole interaction term. The ratios between these components were determined by requiring the asymptotic total ionization cross section and the stopping cross section to agree with the asymptotic form given by the Bethe theory.

The BED model derives the differential and total ionization cross sections for each subshell of the target atom or molecule using the binding energy, average kinetic energy, and the differential oscillator strength of the subshell. One can deduce the required differential oscillator strengths either from reliable photoionization experiments or from accurate theoretical calculations. For atomic ions with an open-shell configuration, we found that a minor modification brought the BED and experimental cross sections into good agreement. For the cases we have tested, the BED cross sections agree very well with experiment from threshold to high incident energies. The BED theory does not contain any fitted or adjustable parameters and is not a perturbation theory. It works well at low incident energies because it is closely linked to the Mott theory, which includes the Coulomb interaction to all orders, although it was derived for a free-electron target.

When details of differential oscillator strengths are not available, we offer the simple binary-encounter-Bethe (BEB) model. This model provides ionization cross sections for targets which are theoretically difficult to handle, e.g., polyatomic molecules. The shape of the differential ionization cross section based on the BEB model may not be realistic, but we found that the corresponding total ionization cross sections are reasonable.

A systematic application of the BED model to more complex targets than those presented here is needed to understand the validity of this simple but remarkably effective model. To do so, not only reliable total photoionization cross sections but also cross sections for each of the outer subshells of atoms and molecules, e.g., those measured using a synchrotron light source, are needed.

Further comparisons of the BED theory with experimental $\sigma_{i}$ of He-like and Li-like ions available in the literature [26,45-47] should reveal whether the use of the $t+1$ denominator is necessary or sufficient to represent the distortion of the incident-electron wave function. Work is in progress to determine reliable differential oscillator strengths for these ions so that the BED model can be applied. Experimental data on the ionization of other one-electron ions would be welcome. Currently, there are 
no experimental SDCS's of one-electron ions to compare with our theory.

With the BED and BEB models, both singly differential and total ionization cross sections can easily be calculated for most atoms, atomic ions, and molecules. The accuracy of electron-impact ionization cross sections provided by the $B E D$ and $B E B$ models over the entire range of energies should be adequate for most applications, including modeling of radiation and plasma effects.

\section{ACKNOWLEDGMENTS}

This research was supported in part by the Office of Fusion Energy of the U.S. Department of Energy (Y.K.K.) and by the National Science Foundation Grant No. PHY9119818 (M.E.R.). We wish to thank M. Inokuti for suggesting the compact expression of Eq. (15), and G. Dunn for providing references to experiments on one-electron ions.

\section{APPENDIX: APPROPRIATE FORM FOR FITTING TOTAL IONIZATION CROSS SECTIONS}

Equation (55) indicates an appropriate functional form of the total ionization cross section (TICS) to be used in fitting the cross section into a compact analytic formula independent of whether the cross section was derived from the BED model or not. For applications in which only total ionization cross sections are needed, the TICS can be fitted to an even simpler function of $t$ than that used in Eq. (55), by avoiding the summation over subshells, using a single $t$ defined by the lowest binding energy $B$, and by omitting $u$.

When a reliable TICS is known--usually through accurate measurements - then a simple form to fit $\sigma_{i}$ is

$$
\sigma_{i}(t)=\frac{4 \pi a_{0}^{2}}{t}\left[a \ln t+b\left(1-\frac{1}{t}\right)+c \frac{\ln t}{t+1}\right],
$$

where $a, b$, and $c$ are fitting parameters. Note that the first term containing $\ln t$ in Eq. (A1) arises from the dipole interaction, while the second $\ln t$ results from integrating the interference term in the Mott formula. If this three-term fit is inadequate, then more terms can be used:

$$
\begin{aligned}
\sigma_{i}(t)= & \frac{4 \pi a_{0}^{2}}{t}\left[a \ln t+b\left(1-\frac{1}{t}\right)+c \frac{\ln t}{t+1}+d \frac{\ln t}{(t+1)^{2}}\right. \\
& \left.+e \frac{\ln t}{(t+1)^{3}}+\cdots\right] .
\end{aligned}
$$

For multishell atoms, $\sigma_{i}$ may require more fitting parameters than for those with simple shell structures. Note that Eq. (A2) can also be adapted to ionizing events that take place without appreciable dipole interactione.g., double ionization of $\mathrm{He}-$ by dropping the $a \ln t$ term but keeping the other $\ln t$ terms.
[1] N.F. Mott, Proc. R. Soc. London Ser. A 126, 259 (1930).

[2] Y.-K. Kim, Radiat. Res. 61, 21 (1975); 64, 96 (1975); 64, 205 (1975).

[3] H. Bethe, Ann. Phys. (Leipzig) 5, 325 (1930).

[4] L. Vriens, in Case Studies in Atomic Physics, edited by E.W. McDaniel and M.R.C. McDowell (North-Holland, Amsterdam, 1969), Vol. 1, p. 335.

[5] See, for instance, J.H. Miller, W.E. Wilson, S.T. Manson, and M.E. Rudd, J. Chem. Phys. 86, 157 (1987), and references therein.

[6] See, for instance, S.P. Khare and W.J. Meath, J. Phys. B 20, 2101 (1987), and references therein.

[7] M. Inokuti, M.A. Dillon, J.H. Miller, and K. Omidvar, J. Chem. Phys. 87, 6967 (1987).

[8] Y.-K. Kim and J.P. Desclaux, Phys. Rev. A 38, 1805 (1988).

[9] M.E. Rudd, Phys. Rev. A 44, 1644 (1991).

[10] Y.-K. Kim, J. Res. Nat. Inst. Stand. Technol. 97, 689 (1992).

[11] L.D. Landau and E.M. Lifshitz, Quantum MechanicsNonrelativistic Theory, 2nd ed. (Addison-Wesley, Reading, MA, 1965), p. 575.

[12] M. Inokuti, Rev. Mod. Phys. 43, 297 (1971).

[13] W.E. Wilson, Radiat. Res. 49, 36 (1972).

[14] Y.-K. Kim, Phys. Rev. A 6, 666 (1972).

[15] H.A. Bethe and E.E. Salpeter, Quantum Mechanics of
One- and Two-Electron Atoms (Springer Verlag, Berlin, 1957), p. 305.

[16] G.H. Wannier, Phys. Rev. 90, 817 (1953).

[17] M.R.H. Rudge, Rev. Mod. Phys. 40, 564 (1968).

[18] C. Froese-Fischer, Comput. Phys. Commun. 64, 431 (1991).

[19] M.E. Rudd, Y.-K. Kim, D.H. Madison, and T.J. Gay, Rev. Mod. Phys. 64, 441 (1992).

[20] J. Berkowitz, Photoabsorption, Photoionization, and Photoelectron Spectroscopy (Academic Press, New York, 1979).

[21] J.W. Gallagher, C.E. Brion, J.A.R. Samson, and F.W. Langhoff, J. Phys. Chem. Ref. Data 17, 9 (1988), and references therein.

[22] Y.-K. Kim and M. Inokuti, Phys. Rev. A 3, 665 (1971).

[23] M. Gryzinski, Phys. Rev. 138, A305 (1965); 138, A322 (1965); 138, A336 (1965).

[24] M.B. Shah, D.S. Elliott, and H.B. Gilbody, J. Phys. B 20, 3501 (1987).

[25] L. Vriens, Proc. Phys. Soc. London 89, 13 (1966).

[26] B. Peart, D.S. Walton, and K.T. Dolder, J. Phys. B 2, 1347 (1969).

[27] P. Defrance, F. Brouillard, W. Claeys, and G. Van Wassenhove, J. Phys. B 14, 103 (1981).

[28] C. Achenbach, A. Müller, E. Salzborn, and R. Becker, J. Phys. B 17, 1405 (1984). 
[29] K. Tinschert, A. Müller, G. Hoffmann, K. Hubert, R. Becker, D.C. Gregory, and E. Salzborn, J. Phys. B 22, 531 (1989).

[30] J. Miller and R.L. Platzman, Proc. Phys. Soc. London A 70, 299 (1957).

[31] Y.-K. Kim, in The Physics of Electronic and Ionic Collisions, edited by J.S. Risley and R. Geballe (Univerisity of Washington Press, Seattle, 1976), p. 741.

[32] T.W. Shyn, Phys. Rev. A 45, 2951 (1992).

[33] K. Omidvar, Phys. Rev. 140, A26 (1965).

[34] S.M. Younger, J. Quant. Spectrosc. Radiat. Transfer 26, 329 (1981).

[35] M.B. Shah, D.S. Elliot, P. McCallion, and H.B. Gilbody, J. Phys. B 21, 2751 (1988).

[36] R.G. Montague, M.F.A. Harrison, and A.C.H. Smith, J. Phys. B 17, 3295 (1984).

[37] C.B. Opal, E.E. Beaty, and W.K. Peterson, At. Data 4, 209 (1972).

[38] R.R. Goruganthu and R.A. Bonham, Phys. Rev. A 34, 103 (1986).

[39] D. Rapp and P. Englander-Golden, J. Chem. Phys. 43, 1464 (1965).

[40] D.R. Schultz, L. Meng, and R.E. Olson, J. Phys. B 25,
4601 (1992).

[41] T.W. Shyn, W.E. Sharp, and Y.-K. Kim, Phys. Rev. A 24, 79 (1981).

[42] R.D. DuBois and M.E. Rudd, Phys. Rev. A 17, 843 (1978).

[43] R.C. Wetzel, F.A. Baiocchi, R.R. Hayes, and R.S. Freund, Phys. Rev. A 35, 559 (1987).

[44] K.W. Hollman, G.W. Kerby III, M.E. Rudd, J.H. Miller, and S.T. Manson, Phys. Rev. A 38, 3299 (1988).

[45] W.C. Lineberger, J.W. Hooper, and E.W. McDaniel, Phys. Rev. 141, 151 (1966).

[46] D.H. Crandall, R.A. Phaneuf, and P.O. Taylor, Phys. Rev. A 18, 1911 (1978).

[47] D.H. Crandall, R.A. Phaneuf, B.E. Hasselquist, and D.C. Gregory, J. Phys. B 12, L249 (1979).

[48] N.Lj. Durić, I.M. Čadež, and M.V. Kurepa, Int. J. Mass Spectrom. Ion Proc. 83, R7 (1988).

[49] M.A. Bolorizadeh and M.E. Rudd, Phys. Rev. A 33, 882 (1986).

[50] J. Schutten, F.J. DeHeer, H.R. Moustafa, A.J.H. Boerboom, and J. Kistemaker, J. Chem. Phys. 44, 3924 (1966). 